\title{
Trajectory Management of Mobile VolP over WiMAX
}

\author{
Salma Rattal \\ EEATI Laboratory \\ Faculty of Sciences and \\ Techniques \\ Hassan 2nd University of \\ Casablanca Morocco
}

\author{
Abdelmajid Badri \\ EEATI Laboratory \\ Faculty of Sciences and \\ Techniques \\ Hassan 2nd University of \\ Casablanca Morocco
}

\author{
Mohammed Moughit \\ National School of Applied \\ Sciences Hassan 1st University \\ Settat \\ Morocco
}

\begin{abstract}
In order to optimize and make network resources more useful, many studies are conducted. However, WiMAX technology researches don't take enough consideration regarding mobility user management.

This paper tackles mobility management of VoIP users over WiMaX. In another way, we propose a new idea to decide the best location of base stations in an enterprise domain according to a daily approximate trajectory of VoIP users.Our research is than organized as follows:Firstly, we watch the users' trajectories on a daily basis then we consider an approximation of users' trajectories.

Secondly, this fictive trajectory is subdivided into small segments. The perpendicular bisectors of the latter segments are used to give a cloud of intersecting points. The same procedure is applied to the obtained cloud until we find a set of points where distances between these points are small enough to be considered as negligible.
\end{abstract}

Finally, the base station location will be where the points are concentrated.

Keywords

VoIP, WiMAX, Mobility, localization.

\section{INTRODUCTION}

Wimax (world wide interoperability for microwave access) is an IEEE 802.16 based technology offering a high quality broadband service, one of its advantages is its coverage area. That's why, this technology is used more and more to carry VoIP (Voice over IP) which is a real time required service and must offer high mobility management.

This paper tackles the following points. The first section provides definitions of VoIP signaling protocols and Wimax technology, which are explained and analyzed separately. The second section shows how the newly proposed system manages the trajectory of VoIP users. This proposal is then configured and simulated into OPNET Modeler in order to study the comparative curves of QoS. The results are discussed in details in the third section. Finally, the conclusion determines the contributions of this technique improving VoIP quality service.

\section{VOIP OVER WIMAX STUDY:}

\subsection{Wimax technology}

The IEEE has evolved through several updates to the standard such as 802.16a, 802.16c, the Fixed WiMAX 802.16d, the mobile $802.16 \mathrm{e}$ set and the $802.16 \mathrm{~m}$. The technology developed the 802.16 in its first version to address line of sight (LOS) access at spectrum ranges from $10 \mathrm{GHz}$ to 66 $\mathrm{GHz}[1]$.
WiMAX is a connection-oriented wide area network. It can potentially provide broadband access to remote places. It can use point-to-multipoint (P2MP) architecture. Its purpose is to deliver broadband seamless quality multimedia services to the end users. A WiMAX base-station can cover almost 3,000 square miles [2].

It provides data rate up to $1 \mathrm{Gbps}$ for a fixed station considering the updates [3]

\subsection{Signaling Protocol SIP}

SIP (Session Initiation Protocol) is an application-layer control protocol that can establish, modify, and terminate multimedia sessions (conferences) such as Internet phone calls. SIP can also invite participants to already-existing sessions, such as multicast conferences. Media can be added to (and removed from) an existing session. SIP transparently supports name mapping and redirection services, which supports personal mobility. The main entities in SIP are: Proxy Server is an intermediary entity that acts as a server and as a client for the purpose of making requests on behalf of other clients. User Agent (UA) is the endpoint entity. [4] User Agents initiate and terminate sessions by exchanging requests and responses. Registrar is a server that updates a location database with the contact information of the user. Redirect Server is a server that maps the SIP address of the called party into zero or new addresses then returns them to the client.

\subsection{VoIP quality service parameters:}

Analyzing the performance of this proposal requires statistical calculations of QOS parameters (MOS, Jitter, end-to-end delay and packet loss).

\subsubsection{End to end delay:}

End-to-end delay consists of the end-system and network delay. The end-system delay occurs due to the encoding and decoding delay and de-jitter buffering delay. Toll quality realtime communication is needed, which limits the maximal tolerable round-trip delay to $200-300 \mathrm{~ms}$; that is, one-way delay must be in the range of $100-150 \mathrm{~ms}$ for adequate performance. [5]

\subsubsection{Jitter}

The end-to-end delay variation between two consecutive packets is called jitter. A jitter of less than $50 \mathrm{~ms}$ is acceptable for high quality VoIP call [6]. If the transmissions delay varies too widely in a VoIP call, the call quality is greatly degraded. [7]

\subsubsection{MOS (Mean Opinion Score):}

The calculated MOS is a standard ETSI and ITU-T. A mathematical calculation of this value could be realized by considering the characteristics of the communication. This recommendation is based on the fact that the damage is added together on a scale of predetermined quality. If a signal goes 
through multiple devices, the equipment damage will add up. [5]

\section{PROPOSED TECHNIQUE:}

\subsection{Approximation of the trajectory:}

During the day, employees are supposed to move along different places of the company. However, using VoIP remains a challenge accordingly.
The purpose of this paper is to propose a new technique to estimate employee's trajectory according to a database that provide daily employee's shifting.

Using this approximation, the location of the base station can be directly calculated.

Figure 1 illustrates this approach with a company architectural model proposed to test the feasibility of this new idea.

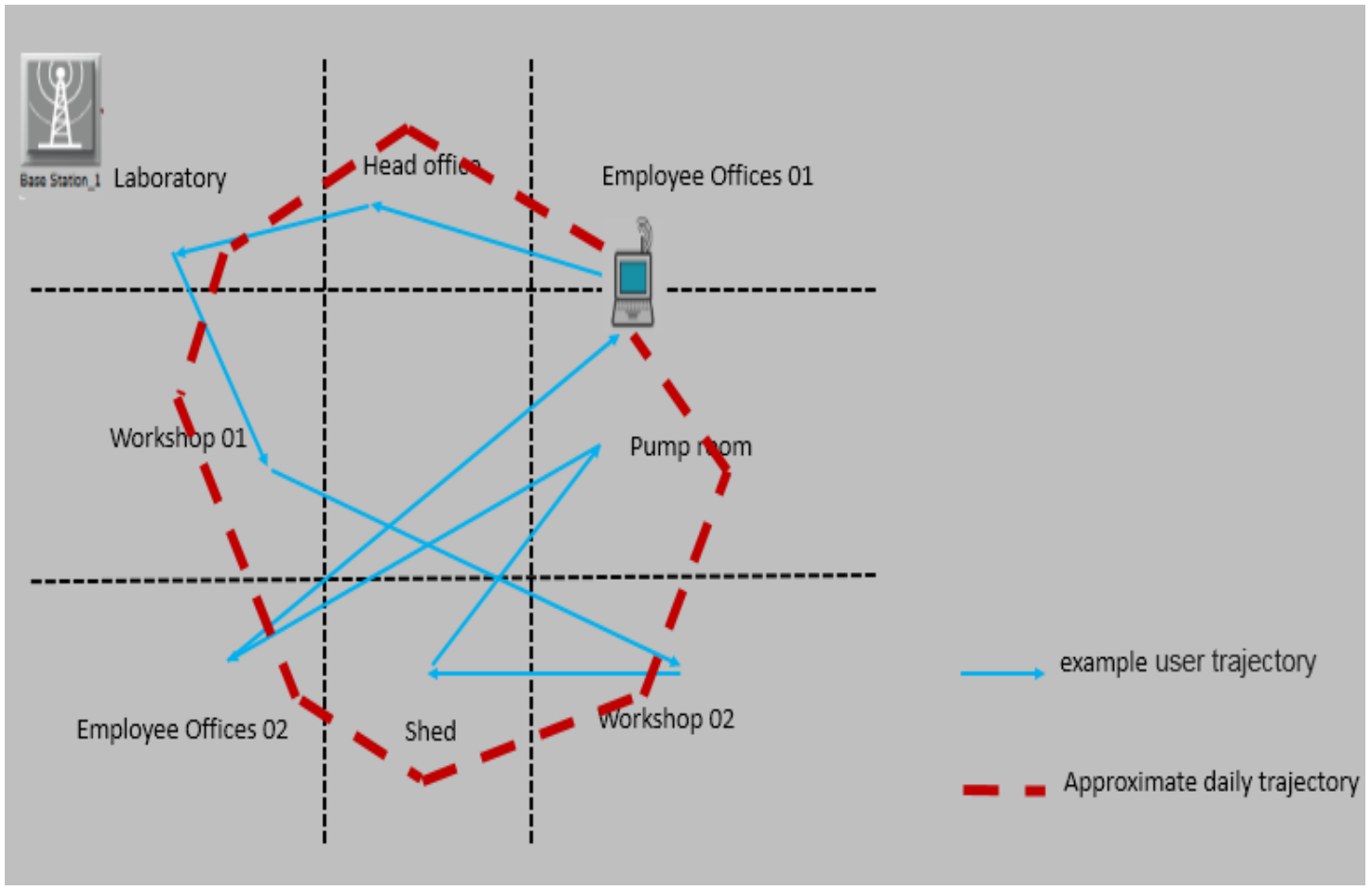

Fig 1: daily trajectory approximation

\subsection{Adopted trajectory:}

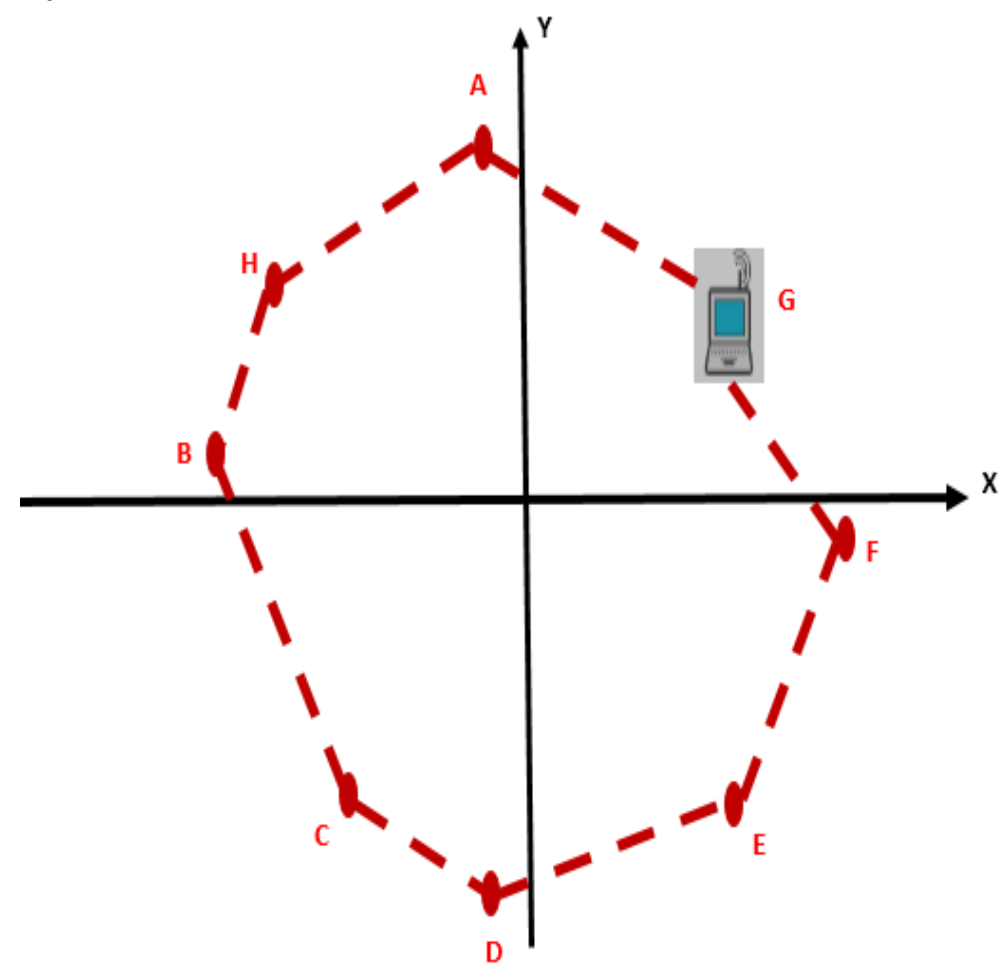

Fig 2: trajectory adopted 
After collecting the data general moving of employees, it's now possible to draw an approximately rounded trajectory as shown in figure 2 .

\subsection{Finding approximatively central points:}

Approximate trajectory is now defined to decide the location of the base station. This technique calculates, in different levels, the central cloud of points which constitutes the base of the next level calculation of another central cloud..

As shown in figure 3 and 4 , the central cloud is found in order to narrow the initial rounded trajectory in different levels until finding the minimum average distance between all points. In this case, the most appropriate location of the base station can be decided accordingly.

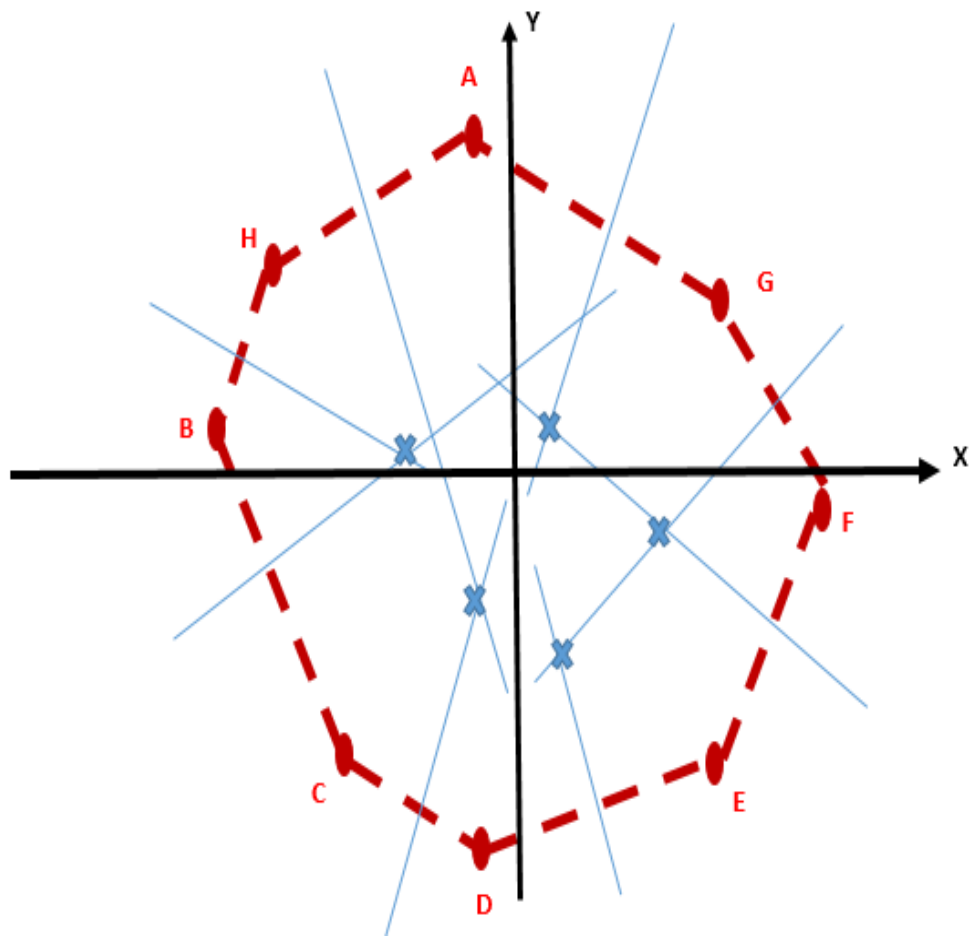

Fig 3: first level calculating central points

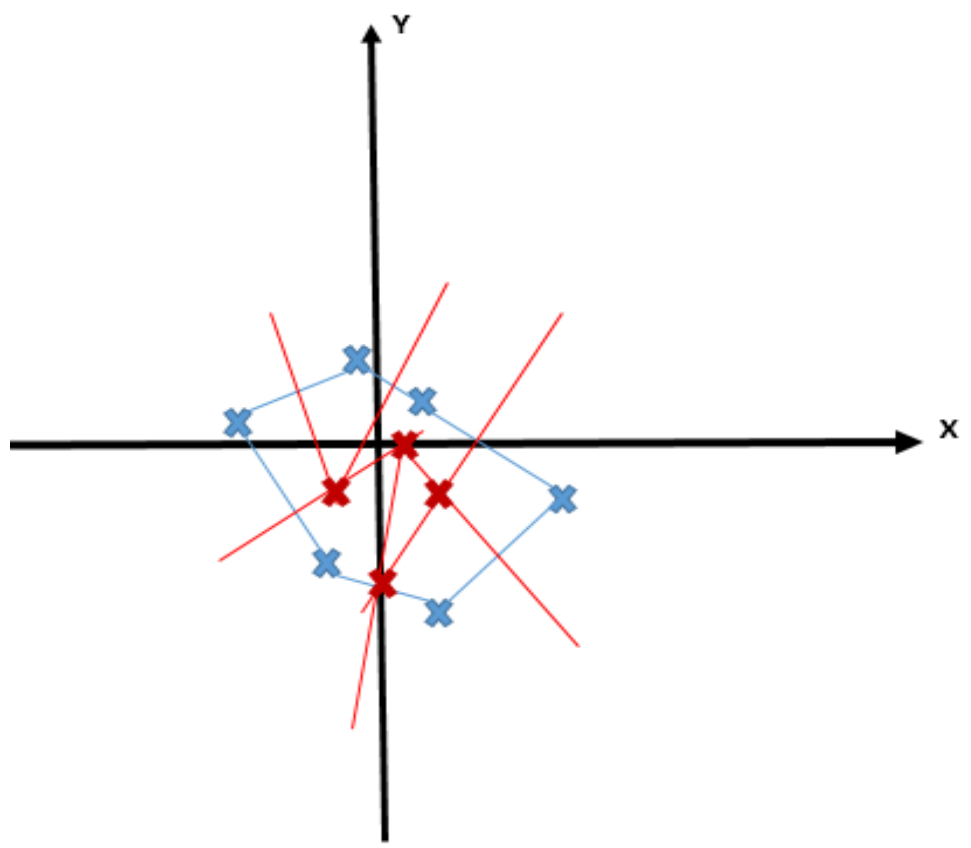

Fig 4: second level calculating central points 


\subsection{The adopted algorithm to implement the proposed technique:}

The proposed technique in the previous section is implemented through setting up an algorithm consisting of operations and instructions of different stages useful for the translation of the explained technique into an effective interpretation.

The algorithm starts with a VoIP over Wimax user trajectory recovery, where employee mobility data is detected and stored in a database to serve in the necessary time. Then the algorithm allows to select a precise number of representative points.

The next step is to retrieve the coordinates of the selected points in order to define the equation of the line joining two consecutive points, and calculate the Equation of the normal of each line passing through the midpoints. After that, the algorithm finds the intersections of the straight lines related to consecutive sides, then retrieves the coordinates of the intersections points and calculate the distances $\mathrm{D}$ between all the consecutive points.

Here, a test must be performed: if the distance D is less than a fixed small $\operatorname{size}^{\varepsilon}$ then the algorithm must choose any single point coordinates of the cloud.

If it is not the case, then retrieve the coordinates of the previous points and redefine the equation of the line joining two consecutive points... until having the distance $\mathrm{D}<\varepsilon$.

Figure 5 defines the adopted algorithm for the successful proposed technique.

\section{ASSESSMENT OF QOS PARAMETERS OF THE SUGGESTED TECHNIQUE}

This section contains the results obtained during the implementation of the algorithm based on calculation of the approximatively found coordinates that are selected at once from the geographic location of the user.

\subsection{Jitter}

In this section, the results of simulating VoIP over WiMAX two scenarios for about a day: the first contains a randomly dispersed number of VoIP users

while the second scenario considers that the geographical location of the base station is found through the implementation of the proposed algorithm.

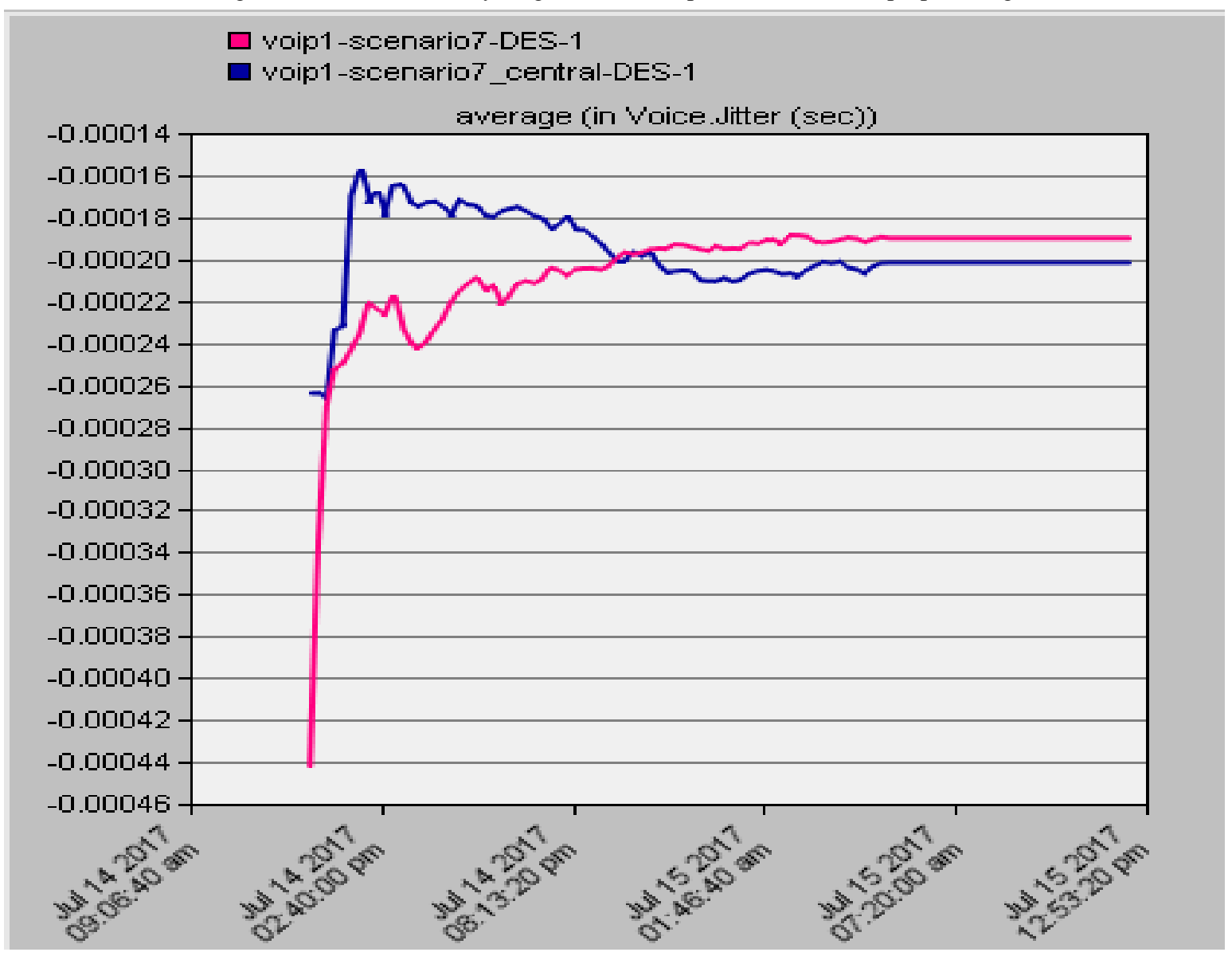

Fig 6: Jitter

It is defined in figure 6 that the fact of integrating the new algorithm allows a change of values of the jitter: at the beginning one will have an increase but later the values stabilize lower throughout the use of the VoIP. 


\subsection{MoS}

As shown in figure 7, the MOS value is equal to 3 for both scenarios. This means that the voice quality is low and must be tackled separately as the MOS score quantifies the quality actually perceived by a certain population. In particular, it takes into account psychoacoustic effects.

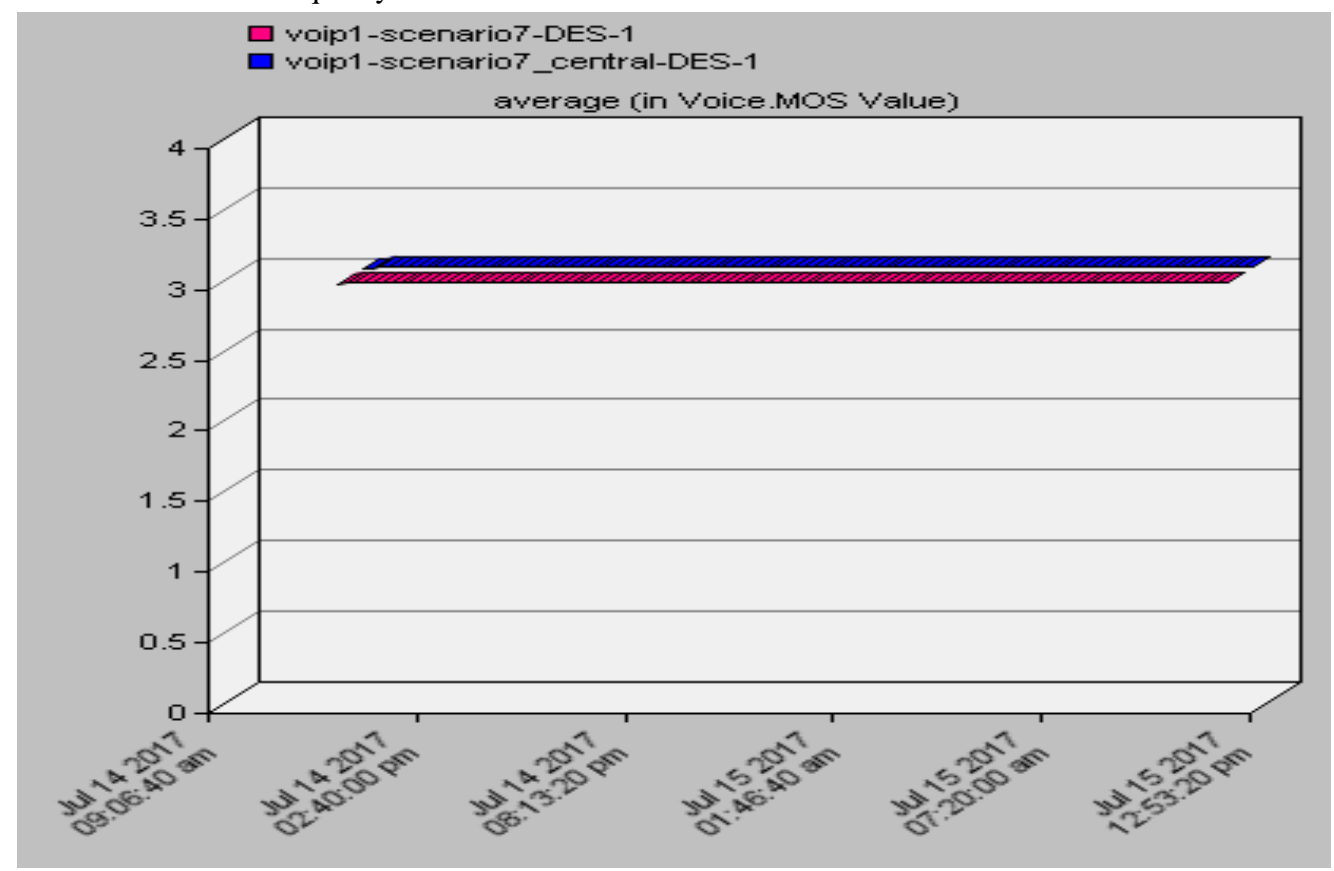

Fig 7: MoS

\subsection{Packet Delay Variation}

In computer networking, packet delay variation (PDV) is the difference in end-to-end one-way delay between selected packets in a flow with any lost packets being ignored.[9]

Figure 8 shows that the packet delay variation is remarkably inferior in the case of the proposed technique.

\subsection{End to End delay}

Figure 9 shows that the scenario taking into consideration the proposed algorithm takes considerably less time comparing to the scenario with randomly distributed users coordinates.

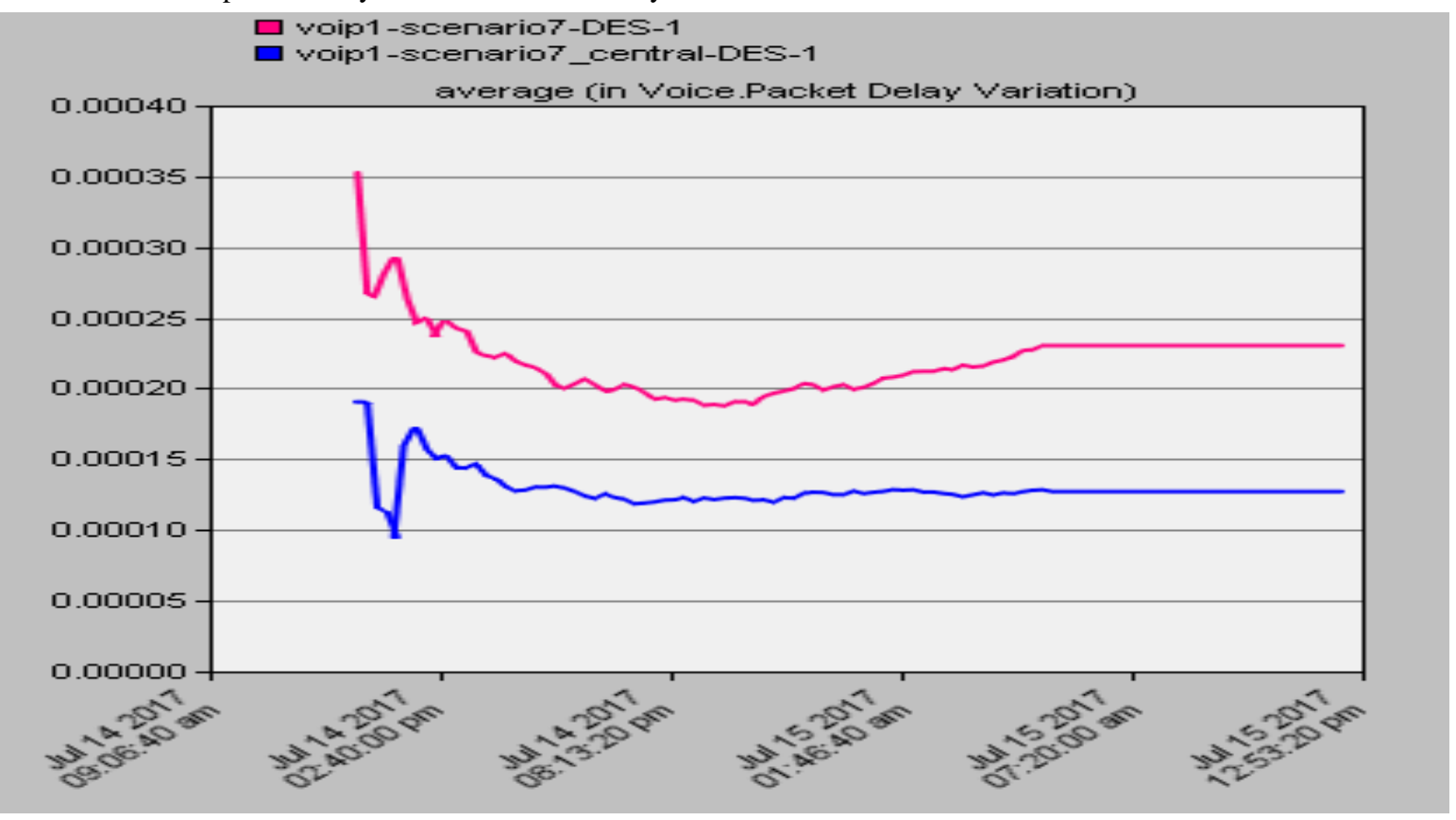

Fig 8: Packet Delay Variation 


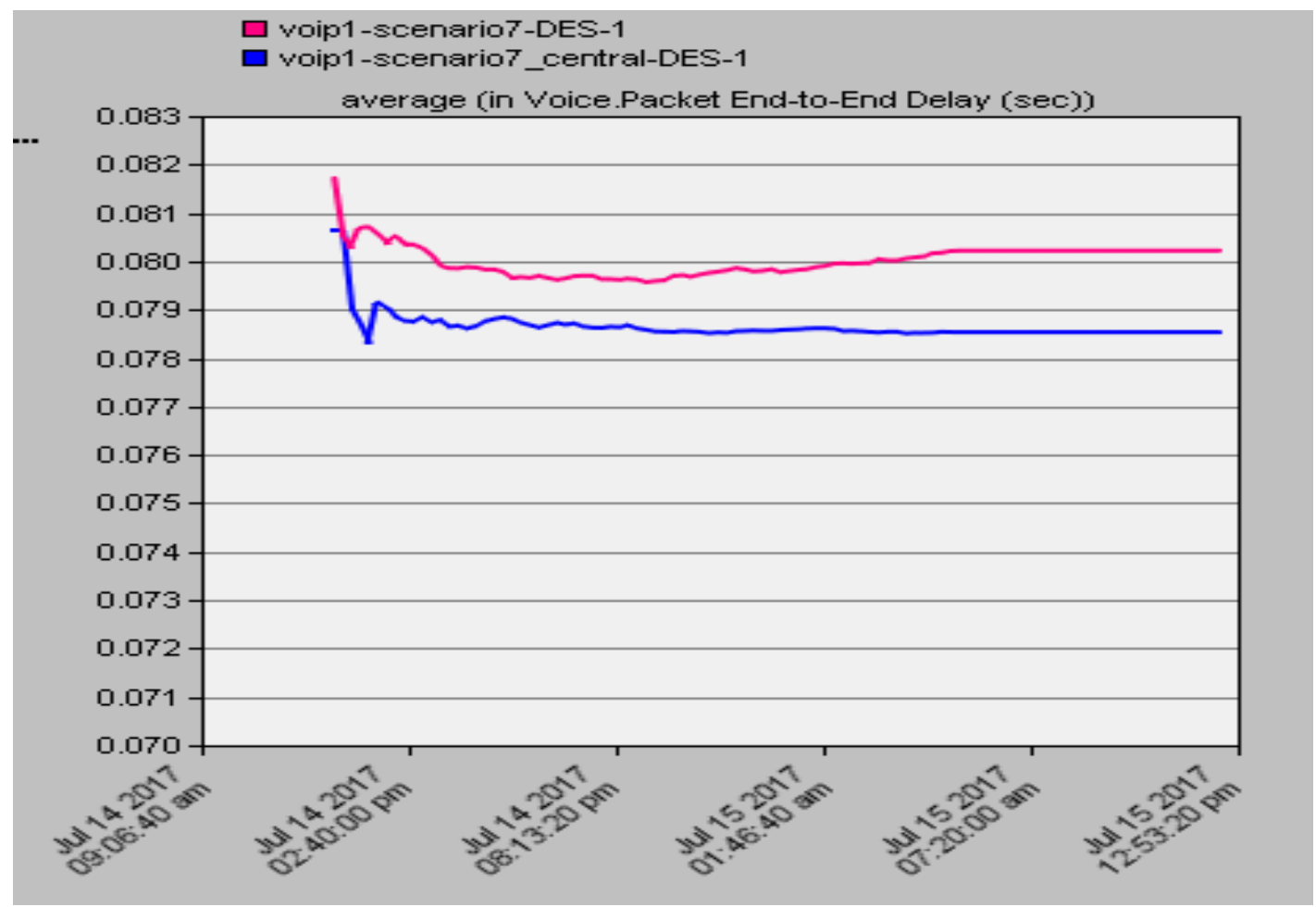

Fig 9: End to End delay

\section{CONCLUSION}

This paper studies the performances the performance obtained when implementing a technique that supports the geographic location of the WiMAX base station in the case of using VoIP.The proposition is of advantage a rather simple but effective technique and has been proved by the results obtained through the parameters of the QoS (Quality of Service).

In the future work, many details will be treated separately: for example the calculation parameters in the proposed algorithm will be treated more accurately, but also the results obtained will take into consideration other applications on IP in parallel with VoIP.

\section{REFERENCES}

[1] WIMAX TECHNOLOGY AND ITS APPLICATIONS Gyan Prakash, Sadhana Pal /International Journal of Engineering Research and Applications (IJERA) ISS : 2248-9622 www.ijera.com Vol. 1, Issue 2, pp.327-336

[2] IEEE 802.16: WiMAX Overview, WiMAX Architecture Mojtaba Seyedzadegan and MohamedOthman International Journal of Computer Theory and Engineering, Vol. 5, No. 5, October 2013
[3] https://en.wikipedia.org/wiki/WiMAX\#cite_noteCarl_Wei nschenk-1

[4] Salma RATTAL, Abdelmajid BADRI and Mohammed MOUGHIT, Performance Analysis of Hybrid Codecs G.711 and G.729 over Signaling Protocols H.323 and SIP. International Journal of Computer Applications 72(3):30-33, ISSN 0975-8887 Published by Foundation of Computer Science, New York, USA. June 2013.

[5] Amit Chhabra Dr. Gurpal Singh "Performance Evaluation and Delay Modelling of VoIP Traffic over 802.11 Wireless Mesh Network" International Journal of Computer Applications (0975 - 8887) Volume 21- No.9, May 2011

[6] Quality of Service Design Overview, Enterprise QoS Solution Reference Network Design Guide CHAPITER 1.

[7] Devarajan Gopal Taye Abdulkadir "Self-Similarity and Internet Performance" Journal of Research and Practice in Information Technology, Vol. 38, No. 2, May 2006.

[8] Twenty-third Annual Joint Conferences of the IEEE Computer and Communications Societies, Volume 2, 7 11 March2004 Page(s):1191 - 1199 vol.2RFC 3393 